\title{
The Prevalence and Replication Capacity of a Tibetan Dominant HBV Strain, C/D Recombinant
}

\author{
Lingyao Du, ${ }^{1}$ Menghan Liu, ${ }^{2}$ Miao Liu, ${ }^{1}$ Taoyou Zhou, ${ }^{1}$ Xing Cheng, \\ Cong Liu, ${ }^{1}$ and Hong Tang ${ }^{1}$ \\ ${ }^{1}$ Center of Infectious Diseases, West China Hospital of Sichuan University, Chengdu 610041, China \\ ${ }^{2}$ Department of Science and Technology, West China Hospital of Sichuan University, Chengdu 610041, China \\ Correspondence should be addressed to Hong Tang; htang6198@hotmail.com
}

Received 20 March 2017; Revised 16 May 2017; Accepted 24 May 2017; Published 21 June 2017

Academic Editor: Jorge F. Quarleri

Copyright (C) 2017 Lingyao Du et al. This is an open access article distributed under the Creative Commons Attribution License, which permits unrestricted use, distribution, and reproduction in any medium, provided the original work is properly cited.

\begin{abstract}
This study aimed to estimate the distribution of hepatitis B virus (HBV) C/D recombinant in Han and Tibet patients with chronic hepatitis B (CHB) and then learn such strain's replication capacity in vivo. A total of 331 serum samples were collected from Han outpatients from Sichuan Province and Tibetan outpatients from Tibet. Viral genotypes in these samples were identified. An HBV replicative plasmid of $\mathrm{C} / \mathrm{D}$ recombinant was constructed with selected genome. Sequentially, HBV replicative mouse models were established and the replication capacity of the viral strain was studied in vivo. In the 314 Han patients, 66\% (207) were infected by genotype B strain while 31\% (96) were by genotype C strain. Only 1\% (3) were by C/D recombinant. In the 17 Tibetan patients, $41 \%$ (7) were by genotype $\mathrm{D}$ and $35 \%$ (6) by C/D recombinant. A plasmid with 1.3 copies of C/D recombinant genome was constructed. And its replication intermediates were found at similar levels to that of genotype $\mathrm{D}$ strain. Thus, $\mathrm{C} / \mathrm{D}$ recombinant, the dominant viral strain in Tibet, was rather rare in the genotype B predominated Han patients from Sichuan Province. And the C/D recombinant replicated at a similar level to viral strain of genotype D in vivo.
\end{abstract}

\section{Introduction}

Decades ago, HBV were subtyped by the amino acid sequences of their surface antigen (HBsAg) [1]. Along with the development of direct sequencing, new method emerged for HBV classification. Phylogenetic analysis of the entire genome has been used for HBV genotyping. Though time consuming and expensive, it is generally accepted as the golden standard [2].

Nine HBV genotypes ranging from A to I and a putative 10th genotype J have been identified by now [3-7]. The intergroup diversity of each genotype is greater than $7.5 \%$ in genomic nucleotide [8]. Viral strains from different genotypes represent unique biological characteristics; these biological characteristics result in various clinical manifestations of the infected patients. Current studies have suggested a causal relationship between HBV genotypes and the spectrum of HBV related diseases, leading us to further investigation in viral genotypes [9-14].

Subgenotypes are evolutionary swarms in genotypes with genomic diversity between $4 \%$ and $7.5 \%$ [14]. However, under such rule, some recombinant strains were misclassified into subgenotypes. Currently, some scientists suggested that the intragenotypic nucleotide divergence between $4 \%$ and $7.5 \%$ was not the best prerequisite to identify subgenotypes $[15,16]$. Even there is traces of recombination in some subgenotypes; recombinant strains should be classified into an independent group when evidence of combination was confirmed and the genetic divergence fell into the classical definition of subgenotypes [17]. Based on that, more than 30 recombinant strains have been reported [18]. The recombination regions were always the essential functioning regions affecting viral biological characteristics, and these characteristics lead to various manifestations in patients $[19,20]$.

Geographic environment and genetic background contribute to the development of genotypes, subgenotypes, and recombinants [21]. In Tibet, the summit of Asian continent, HBV infection is highly prevalent in patients. In 2002, local doctors conducted a viral sequence analysis with over 1000 serum samples from asymptomatic chronic HBV carriers. The sequencing result showed highly homologous sequences, 
implying the existence of a dominant strain. Phylogenetic analysis in its $S$ gene identified it as genotype $D$, but it lacked the typical nucleotide loss in nt2855-2887. Full genome analysis classified it into genotype C. Such results strongly suggested that the dominant strain in Tibet is a new recombination. Later, a representative genome was acquired. Full genome as well as each open reading frame was analyzed with the 23 standard sequences from genotype $\mathrm{A}$ to $\mathrm{F}$ in NCBI. The result showed a region around nt50-1540 covering preS2/S region and part of the $\mathrm{P}$ region from genotype $\mathrm{D}$ was integrated into genotype $\mathrm{C}$ to form the recombinant viral strain [22].

Reports on C/D recombinant followed the previous finding. A study in Uygur from Shinkiang found that the $\mathrm{C} / \mathrm{D}$ recombinant was epidemic in 109 chronic $\mathrm{HBV}$ carries, with a ratio of $29.4 \%(N=32)$ [23]. Another study in 2009 confirmed that the C/D recombinant was dominant in both Shinkiang and Tibet [24]. However, the distribution of the viral strain in Han nationality remains unknown. And relationship between viral biological characteristics and the minority population susceptibility in west China needs further investigation.

Replicative HBV plasmid is applicable to study the viral biological characteristics. Such plasmid can replicate effectively both in vitro and in vivo. It contains 1.3 copies of $\mathrm{HBV}$ genome with $4.1 \mathrm{~Kb}$ in length in its multiple cloning sites. Besides $3.2 \mathrm{~Kb}$ of the $\mathrm{HBV}$ genome, a $0.9 \mathrm{~Kb}$ of the repeating sequence from upstream Enhance I and X promoter to downstream Poly A is integrated. Thus the plasmid usually named pHBV4.1 [25].

In this study, we aimed to roughly estimate the distribution of HBV C/D recombinant in Han nationalities in Sichuan Province, West China, as well as Tibetan patients in Tibet. We also aimed to first identify the special viral strain and then to extract it to construct a replicative plasmid pHBV4.1(C/D). By doing so, we hoped to understand the biological characteristics of such special HBV strain.

\section{Results}

2.1. Distribution of C/D Recombinant Strain in Serum Samples from Different Groups. In this study, we collected a total of 331 serum samples, and five genotypes were identified. 210 samples were identified as genotype B. 97 samples were identified as genotype $\mathrm{C}$, and 7 samples were identified as genotype $\mathrm{D}$. $\mathrm{B} / \mathrm{C}$ recombinant strain and $\mathrm{C} / \mathrm{D}$ recombinant strain were found in 8 and 9 samples, respectively.

Based on the patients' ethnic background, the samples were divided into two groups: the Han group and the Tibetan group. In the Han group: 314 serum samples were collected from Han $\mathrm{CHB}$ patients from Sichuan Province; and 4 viral strains were detected; genotype B strain infected 207 (66\%) patients, genotype C strain infected 96 (31\%) patients, B/C recombinant strain infected $8(2 \%)$ patients, and C/D recombinant strain infected only $3(1 \%)$ patients. In the Tibetan group: 17 serum samples were collected from Tibetan $\mathrm{CHB}$ patients from Tibet, and also 4 viral strains were detected; genotype B strain infected 3 (18\%) patients, genotype C strain infected $1(6 \%)$ patient, genotype D strain infected 7 (41\%) patients, and C/D recombinant strain infected 6 (35\%) patients. Moreover, the viral load, composition of BCP mutations and liver stiffness also showed significant differences in the Han group and the Tibetan group. Composition of each virus strain in these two groups and other information were shown in Table 1.

To learn the unique clinical manifestation or virological features of $\mathrm{C} / \mathrm{D}$ recombinant, these data in $\mathrm{C} / \mathrm{D}$ recombinant infected patients were analyzed and compared with that of all patients. As mentioned before, 9 patients were confirmed to be infected with $\mathrm{HBV} \mathrm{C} / \mathrm{D}$ recombinant in total. It turned out that alcohol-abuse rate was higher in $\mathrm{C} / \mathrm{D}$ recombinant infected patients as well as the occurrence rate of BCP A1762T/G1764A double mutation. The detailed clinical information was listed in Table 2.

\subsection{Construction of HBV Replicative Plasmid pHBV4.1(C/D).} One Tibetan serum sample which was infected by $\mathrm{C} / \mathrm{D}$ recombinant strain showed high viral load. It was selected for DNA extraction and was then used as PCR template. With a specially designed primer pair, HBV full genomic DNA was acquired. Sequentially two fragments of $1.7 \mathrm{~Kb}$ and $2.4 \mathrm{~Kb}$ were successfully acquired, ligated, and inserted. Then one germ of clone containing newly constructed plasmid was picked out for confirmation. The result was that a fragment about $4.1 \mathrm{~Kb}$ could be digested out from the plasmid and full $\mathrm{HBV}$ genomic DNA about $3.2 \mathrm{~Kb}$ could be amplified from the recombinant plasmid for sequence confirmation. The related gel bands were showed in Figure 1. Amplification product was sequenced in SinoGenoMax Co., Ltd. (Beijing). The numbering of the HBV genome started with TTCC, namely, the restriction site of EcoR I (GGAATTC), downstream of the initiation codon (ATG) of preS2 region. In some strains with mutations, it started with CTCC. Phylogenetic analysis showed the sequence belonged to a branch next to genotype $\mathrm{C}$ (Figure 2). In the subsequent recombination analysis, a fragment (nt1-1480) covering preS2/S region and $\mathrm{X}$ region of the selected viral strain showed higher similarity to reference sequence of genotype $\mathrm{D}$ than the similarity to reference sequences of other genotypes including genotype $\mathrm{C}$ (Figure 3 ). This proved that the newly constructed plasmid was truly derived from the $\mathrm{C} / \mathrm{D}$ recombinant.

2.3. Establishment of the Hydrodynamic $H B V$ Replicative Mouse Model with pHBV4.1(C/D) and Study of Its Replication Capacity. Two groups of hydrodynamic HBV replicative mouse models were established with pHBV4.1(D) and pHBV4.1(C/D). The mouse model of pHBV4.1(D) was established and verified in our lab previously. It was confirmed to be efficient for learning HBV replication, transcription, and replication. Thus the replication capacity of pHBV4.1(C/D) could be studied in a similar model established according to the same procedures. With the same primer pair and reaction condition, full HBV genomic DNA in mouse serum was amplified. Then the amplified products were sequenced again. After BLAST, the relevant HBV sequences with an identity over $97 \%$ all belonged to $\mathrm{C} / \mathrm{D}$ recombinant. The result was the same as the sequencing result after plasmid was successfully constructed. 
TABLE 1: Distribution of HBV genotypes in enrolled Han patients and Tibetan patients.

\begin{tabular}{|c|c|c|c|c|}
\hline \multirow{2}{*}{ Items } & \multicolumn{2}{|c|}{ Statistical description percentage (amount)/median (interquartile range) } & \multirow{2}{*}{ Statistical value } & \multirow{2}{*}{$p$ value } \\
\hline & Han patients $(N=314)$ & Tibetan patients $(N=17)$ & & \\
\hline Genotype & $\begin{array}{l}\text { B } 65.92 \%(N=207) \\
\text { C } 30.57 \%(N=96) \\
\text { B/C } 2.55 \%(N=8) \\
\text { C/D } 0.96 \%(N=3)\end{array}$ & $\begin{array}{c}\text { B } 17.65 \%(N=3) \\
\text { C } 5.88 \%(N=1) \\
\text { D } 41.18 \%(N=7) \\
\text { C/D } 35.29 \%(N=6)\end{array}$ & Fisher & $<0.001$ \\
\hline Gender & $\begin{array}{l}\text { Male } 84.08 \%(N=264) \\
\text { Female } 15.92 \%(N=50)\end{array}$ & $\begin{array}{l}\text { Male } 82.35 \%(N=14) \\
\text { Female } 17.65 \%(N=3)\end{array}$ & $\chi^{2}=0.000$ & 1 \\
\hline Age & $33.5(15)$ & $38(22.5)$ & $Z=-1.019$ & 0.308 \\
\hline Alcohol & $\begin{array}{l}\text { Alcohol-abuse } 18.47 \%(N=58) \\
\text { Alcohol-free } 81.53 \%(N=256)\end{array}$ & $\begin{array}{l}\text { Alcohol-abuse } 35.29 \%(N=6) \\
\text { Alcohol-free } 64.71 \%(N=11)\end{array}$ & $\chi^{2}=1.947$ & 0.1629 \\
\hline Family history & $\begin{array}{c}\text { Positive } 39.17 \%(N=123) \\
\text { Negative } 60.83 \%(N=191)\end{array}$ & $\begin{array}{c}\text { Positive } 58.82 \%(N=10) \\
\text { Negative } 41.18 \%(N=7)\end{array}$ & $\chi^{2}=1.838$ & 0.1752 \\
\hline ALT (IU/L) & $40(41)$ & $38(34.5)$ & $Z=0.286$ & 0.388 \\
\hline TB (umol/L) & $12.50(9.44)$ & $12.82(6.76)$ & $Z=-0.242$ & 0.596 \\
\hline $\operatorname{ALB}(g / L)$ & $50.70(23.60)$ & $47.50(8.20)$ & $Z=1.627$ & 0.104 \\
\hline ALP (IU/L) & $91.50(42)$ & $93(34)$ & $Z=-0.213$ & 0.831 \\
\hline GGT (IU/L) & $49(50)$ & $45(41)$ & $Z=0.483$ & 0.629 \\
\hline $\operatorname{AFP}(\mathrm{ng} / \mathrm{mL})$ & $3.46(4.25)$ & $3.06(5.02)$ & $Z=0.379$ & 0.705 \\
\hline HBeAg & $\begin{array}{l}\text { Positive } 66.56 \%(N=209) \\
\text { Negative } 33.44 \%(N=105)\end{array}$ & $\begin{array}{l}\text { Positive } 76.47 \%(N=13) \\
\text { Negative } 23.53 \%(N=4)\end{array}$ & $\chi^{2}=0.33859$ & 0.5606 \\
\hline $\log 10 \mathrm{HBVDNA}(\mathrm{IU} / \mathrm{mL})$ & $7.27(3.21)$ & $6.13(1.60)$ & $Z=3.525$ & $<0.001$ \\
\hline BCP mutation & $\begin{array}{c}\text { A1762T/G1764A 16.56\% }(N=52) \\
\text { G1896A 16.56\% }(N=52) \\
\text { A1762T/G1764A/G1896A } 2.55 \%(N=8) \\
\text { Others 3.50\% }(N=11) \\
\text { Not detected } 60.83 \%(N=191)\end{array}$ & $\begin{array}{c}\text { A1762T/G1764A 41.18\% }(N=8) \\
\text { G1896A 5.88\% }(N=1) \\
\text { A1762T/G1764A/G1896A 5.88\% }(N=1) \\
\text { Not detected 41.18\% }(N=7)\end{array}$ & Fisher & 0.02899 \\
\hline Fibroscan (Kpa) & $8.00(6.20)$ & $10.45(5.18)$ & $Z=-2.499$ & 0.012 \\
\hline
\end{tabular}

Viral replication intermediates were isolated according to previously published method [26]. And with introduction of DNase digestion during the procedures, the input plasmid DNA was removed and the isolated nucleotides were purified replication intermediates. Three independent experiments were carried out and totally six mice were modeled in each experiment: 3 in the pHBV4.1(D) group and 3 in the pHBV4.1(C/D) group. Results showed that the HBV replicative intermediates could be detected by DNA filter hybridization in each group. The semiquantitative data captured by Quantity One showed that $\mathrm{pHBV} 4.1(\mathrm{C} / \mathrm{D})$ possessed similar replication capacity of pHBV4.1(D), demonstrating no significant differences (0.87 versus $1.00 p=0.9751)$. It implied that newly constructed plasmid could replicate effectively and stably in mouse hepatocytes. Viral strain of $\mathrm{C} / \mathrm{D}$ recombinant strain presented similar replication capacity as viral strain of genotype D (Figure 4).

\section{Discussion}

Geographically, HBV genotypic distribution in China is that genotype $\mathrm{C}$ is predominant in the north while genotype $\mathrm{B}$ is prevalent in the south. We found such genotypic distribution consistent in Han patients from Sichuan Province of Southwest China. The predominant strains in Sichuan
Province were genotypes B and C, and genotype B was the majority. In the 314 Sichuan Han patients, only 3 patients were confirmed to be with $\mathrm{C} / \mathrm{D}$ recombinant strain infection. But when it came to Tibetan patients, the situation was quite different. From the limited serum samples collected from Tibetan patients enrolled in the study, genotype $\mathrm{D}$ and $\mathrm{C} / \mathrm{D}$ recombinant strains were dominant. There were $76 \%$ $(N=13)$ of Tibetan patients infected by these two viral strains, and $46 \%(N=6)$ were confirmed to be infected by $\mathrm{C} / \mathrm{D}$ recombinant strain. The genotype $\mathrm{D}$ strain seemed to infect more patients $(N=7)$ than $\mathrm{C} / \mathrm{D}$ recombinant in the study. This result is different from the previous report [24]. The reason could be that all C/D recombinant strain shared the same sequence similar to the $S$ region of genotype $D$ viral strain. So when $S$ gene sequencing was used for genotype identification, $\mathrm{C} / \mathrm{D}$ recombinants could be mistaken as genotype D. Moreover, there was an isolate of HBV $\mathrm{C} / \mathrm{D}$ recombinant with a deficiency of nucleotides between nt2853 and nt2855. And a repeating sequence of seven nucleotides (GCATGGG) located upstream and downstream of the lack, respectively. Such location and genomic structure perfectly mimic that of the genotype $\mathrm{D}$ viral strain which located between nt2855 and nt2887. This strain could also be mistaken as genotype $\mathrm{D}$. Our results demonstrated that $\mathrm{C} / \mathrm{D}$ recombinant of the $\mathrm{HBV}$ viral strain was a predominant $\mathrm{HBV}$ 
TABLE 2: The clinical manifestations and virological features of C/D recombinant.

\begin{tabular}{|c|c|c|c|c|}
\hline \multirow{2}{*}{ Items } & \multicolumn{2}{|c|}{ Statistical description percentage (amount)/median (interquartile range) } & \multirow{2}{*}{ Statistical value } & \multirow{2}{*}{$p$ value } \\
\hline & $\mathrm{C} / \mathrm{D}$ recombinant infected patients $(N=9)$ & All patients $(N=331)$ & & \\
\hline Ethnicity & $\begin{array}{c}\text { Tibetan } 66.67 \%(N=6) \\
\operatorname{Han} 33.33(N=3)\end{array}$ & $\begin{array}{l}\text { Tibetan } 5.14 \%(N=17) \\
\text { Han } 94.86 \%(N=314)\end{array}$ & Fisher & $<0.001$ \\
\hline Gender & $\begin{array}{c}\text { Male } 77.78 \%(N=7) \\
\text { Female } 22.22 \%(N=2)\end{array}$ & $\begin{array}{l}\text { Male } 81.87 \%(N=271) \\
\text { Female } 18.13 \%(N=60)\end{array}$ & $\chi^{2}=0.000$ & 1 \\
\hline Age & $40(22.5)$ & $33.50(15)$ & $Z=0.864$ & 0.388 \\
\hline Alcohol & $\begin{array}{l}\text { Alcohol-abuse } 44.44 \%(N=4) \\
\text { Alcohol-free } 55.56 \%(N=5)\end{array}$ & $\begin{array}{l}\text { Alcohol-abuse } 82.48 \%(N=273) \\
\text { Alcohol-free } 17.52 \%(N=58)\end{array}$ & $\chi^{2}=6.0651$ & 0.01379 \\
\hline Family history & $\begin{array}{c}\text { Positive } 77.78 \%(N=7) \\
\text { Negative } 22.22 \%(N=2)\end{array}$ & $\begin{array}{c}\text { Positive } 39.58 \%(N=131) \\
\text { Negative } 60.42 \%(N=200)\end{array}$ & $\chi^{2}=3.8364$ & 0.05015 \\
\hline ALT (IU/L) & $38(32)$ & $40(42)$ & $Z=-0.977$ & 0.328 \\
\hline TB (umol/L) & $11.50(7.41)$ & $12.40(9.44)$ & $Z=-0.611$ & 0.542 \\
\hline $\operatorname{ALB}(\mathrm{g} / \mathrm{L})$ & $47.50(9.10)$ & $50.80(22.40)$ & $Z=-1.083$ & 0.278 \\
\hline ALP (IU/L) & $79(33.25)$ & $91(42.25)$ & $Z=-1.207$ & 0.228 \\
\hline GGT (IU/L) & $45.50(34.50)$ & $46(54)$ & $Z=-0.049$ & 0.962 \\
\hline $\operatorname{AFP}(\mathrm{ng} / \mathrm{mL})$ & $3.55(6.27)$ & $3.42(3.54)$ & $Z=0.069$ & 0.944 \\
\hline HBeAg & $\begin{array}{l}\text { Positive } 77.78 \%(N=7) \\
\text { Negative } 22.22 \%(N=2)\end{array}$ & $\begin{array}{l}\text { Positive } 68.28 \%(N=226) \\
\text { Negative } 31.72 \%(N=105)\end{array}$ & $\chi^{2}=0.058456$ & 0.809 \\
\hline $\log 10 \mathrm{HBVDNA}(\mathrm{IU} / \mathrm{mL})$ & $6.69(1.62)$ & $7.27(3.04)$ & $Z=-1.125$ & 0.26 \\
\hline BCP mutation & $\begin{array}{c}\text { A1762T/G1764A 44.44\% }(N=4) \\
\text { G1896A 11.11\% }(N=1) \\
\text { A1762T/G1764A/G1896A } 11.11 \%(N=1) \\
\text { Not detected 33.33\% }(N=3)\end{array}$ & $\begin{array}{c}\text { A1762T/G1764A 16.01\% }(N=53) \\
\text { G1896A 18.13\% }(N=60) \\
\text { A1762T/G1764A/G1896A 3.02\% }(N=10) \\
\text { Others 3.02\% }(N=10) \\
\text { Not detected } 59.82 \%(N=198)\end{array}$ & Fisher & 0.03049 \\
\hline Fibroscan (Kpa) & $11.5(8.55)$ & $7.95(6.42)$ & $Z=1.412$ & 0.921 \\
\hline
\end{tabular}

strain in Tibetan patients from Tibet and rarely seen in Han patients from Southwest China.

In 1999, Mayerat et al. theorized that HBV genetic divergence would affect the HBV related diseases' spectrum. Mayerat et al. not only proved that genotype A virus was related to chronic infection, but also for the first time related the genotype D virus to acute transformation of the disease [9]. This study brought attention to the correlation between HBV genetic divergence and clinical characteristics in patients and marked the start of various studies on this topic [10-13]. Various studies later all confirmed that viral genetic divergence could lead to the variation in clinical manifestation and prognosis of patients. However, current studies about C/D recombinant mainly focused on sequence study, distribution, and some other epidemiological characteristics. Data about the recombinant's biological characteristics is lacking, not to mention the clinical features of those infected patients. An effectively replicative plasmid would be helpful to solve the problem. In our study, the $\mathrm{HBV}$ replicative plasmid of $\mathrm{C} / \mathrm{D}$ recombinant strain was successfully constructed. Thus HBV replicative animal models of this strain could be established for studies on viral replication, pathogenesis, and drug resistance afterwards.

Previously, the plasmid with 1.3 copies of $\mathrm{HBV}$ genome had been proved to replicate and transcribe stably as the natural $\mathrm{HBV}$ in hepatocytes in both transgenic mice and regular BALB/C mice $[25,26]$. It is promising that the newly constructed plasmid with the same structure would replicate efficiently. And It showed similar replication capacity to pHBV4.1(D). For the first time, the replicative characteristics of the $\mathrm{HBV} \mathrm{C} / \mathrm{D}$ recombinant were studied. We adopted the genotype $\mathrm{D}$ strain as the control to assess the $\mathrm{C} / \mathrm{D}$ recombinant not only for the proved stable replicative ability of genotype $\mathrm{D}$, but also for its contribution of a recombinant region for the $\mathrm{C} / \mathrm{D}$ recombinant. The fact that no significant difference was found may be resulting from the same $S$ region and serotype (ayw) of the two strains. But learning the differences of $\mathrm{HBV}$ C/D recombinant from genotype D strain could still be a good start of further investigations.

The unique genome of $\mathrm{HBV} \mathrm{C} / \mathrm{D}$ recombinant was suggested to influence the patients' manifestation. Previous study also revealed that $\mathrm{C} / \mathrm{D}$ recombinant exhibited higher frequency with $\mathrm{HBeAg}$ positive, high level of HBV DNA, and BCP A1762T/G1764A double mutation [27]. In our study, we found similar result in BCP mutation. The composition of BCP mutations showed significant difference between $\mathrm{C} / \mathrm{D}$ recombinant group and the entire group (Fisher, $p=$ 0.03049 ). The percentage of A1762T/G1764A double mutation in $\mathrm{C} / \mathrm{D}$ recombinant was especially higher than the entire group (44.44\% versus $16.01 \%)$. However, we did not observe any differences in the HBeAg positive frequency $(p=0.089)$ and HBV DNA load ( $p=0.26$ ) between C/D recombinant group and the entire groups. The samples size might account for this and further study remains to proceed. 


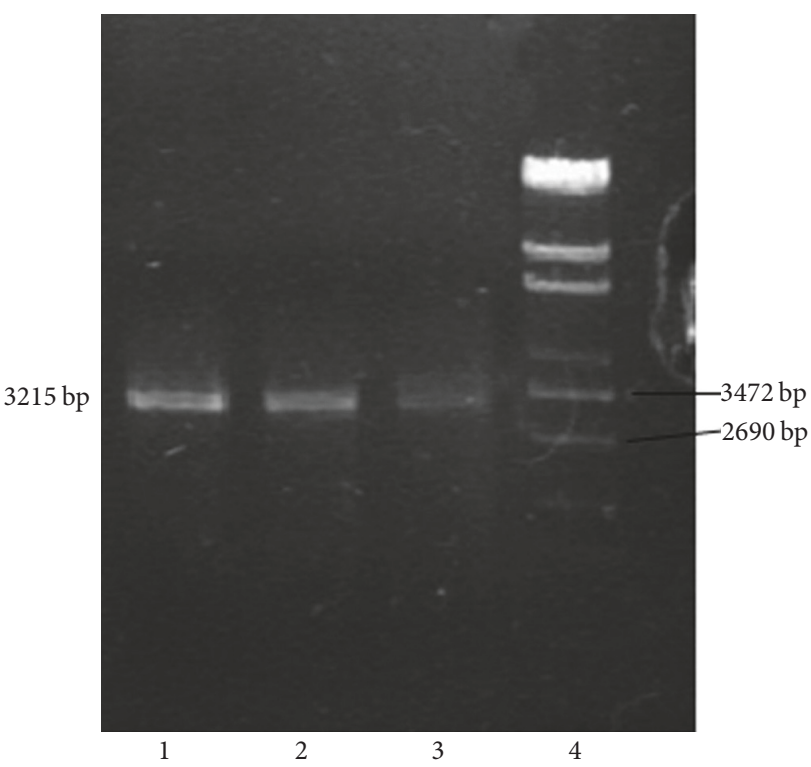

(a)

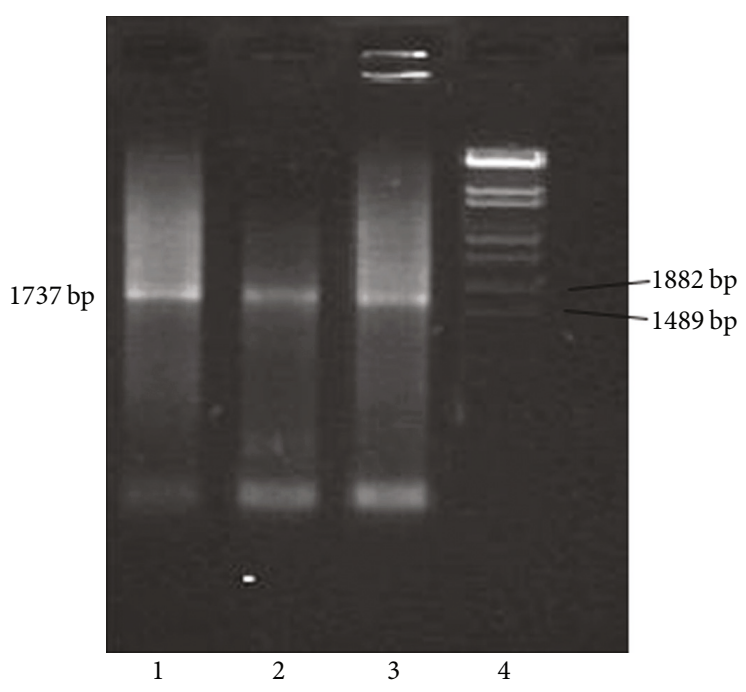

(c)

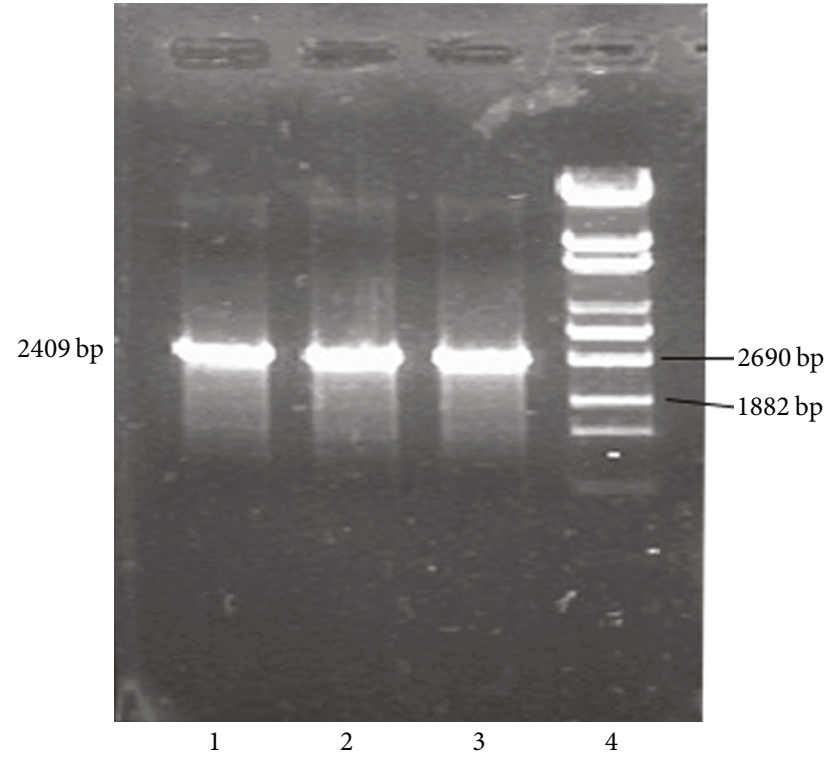

(b)

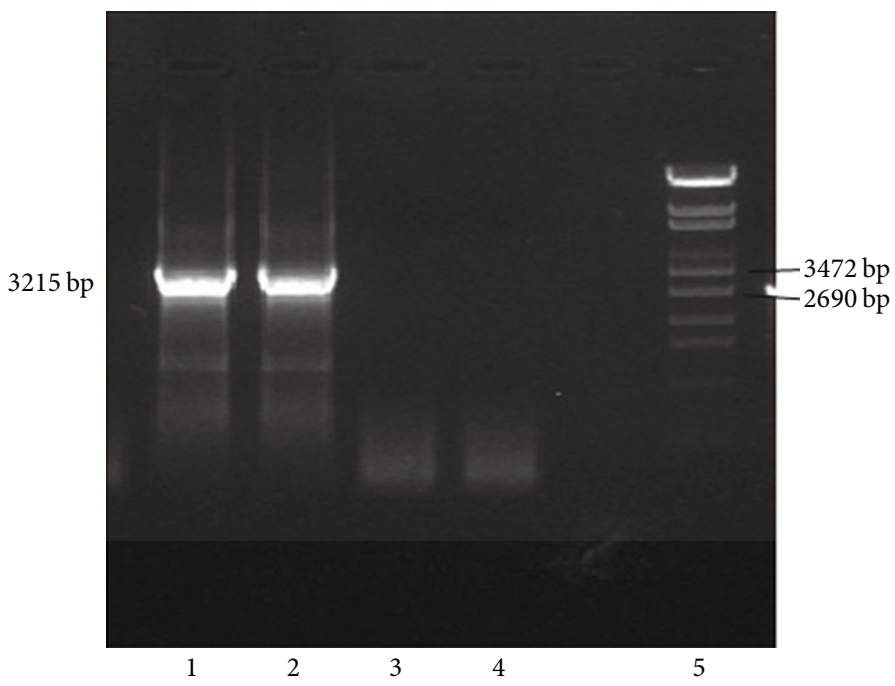

(d)

FIgURE 1: Gel bands during the construction procedures of HBV replicative plasmid of C/D recombinant. (a) Amplification of HBV full genome with templates extracted from serum samples. Lines 1-3 were amplification products. Line 4 was $\lambda$ DNA/EcoT14 digested marker. (b) Amplification of HBV fragment of $2.4 \mathrm{~Kb}$ with amplified and ligated HBV genomic DNA as templates. Lines 1-3 were amplification products. Line 4 was $\lambda$ DNA/EcoT14 digested marker. (c) Amplification of HBV fragment of $1.7 \mathrm{~Kb}$ with amplified and ligated HBV genomic DNA as templates. Lines 1-3 were amplification products. Line 4 was $\lambda$ DNA/EcoT14 digested marker. (d) Amplification of HBV full genome with newly constructed replicative plasmid. The plasmid was derived from ligation of $1.7 \mathrm{~Kb}$ and $2.4 \mathrm{~Kb}$ fragments and vector pUC18. After transfection and selection, positive DH5 $\alpha$ clones were acquired for plasmid extraction and full viral genome amplification was conducted for verification. Lines 1 and 2 were amplification products with selected plasmid as template. Lines 3 and 4 were amplification products with only vector pUC18 as templates. Line 5 was $\lambda \mathrm{DNA} / \mathrm{EcoT} 14$ digested marker.

Moreover, according to a previous study in Fujian province, China, genotype $\mathrm{C}$, genotype $\mathrm{D}$, and their recombinant were identified in patients with HBV associated hepatocellular carcinoma (HBV-HCC). Though the pathogenesis of $\mathrm{C} / \mathrm{D}$ recombinant remains to be clarified and clinical manifestations of those infected patients need more data, we could still make a sidewise approach through genotype C and D strains. Two large scale clinical trials in Hong Kong and Taiwan confirmed that patients infected by genotype $\mathrm{C}$ strain encounter a higher risk of HBV-HCC genesis. And two studies in India and Iran showed that genotype D strain infection was related to higher histological inflammation and higher risk of HBV-HCC genesis [10, 11, 13]. Therefore, the C/D recombinant may have similar pathogenicity to these two strains (genotype $\mathrm{C}$ and genotype $\mathrm{D}$ ). More investigations should be implemented to verify it. 


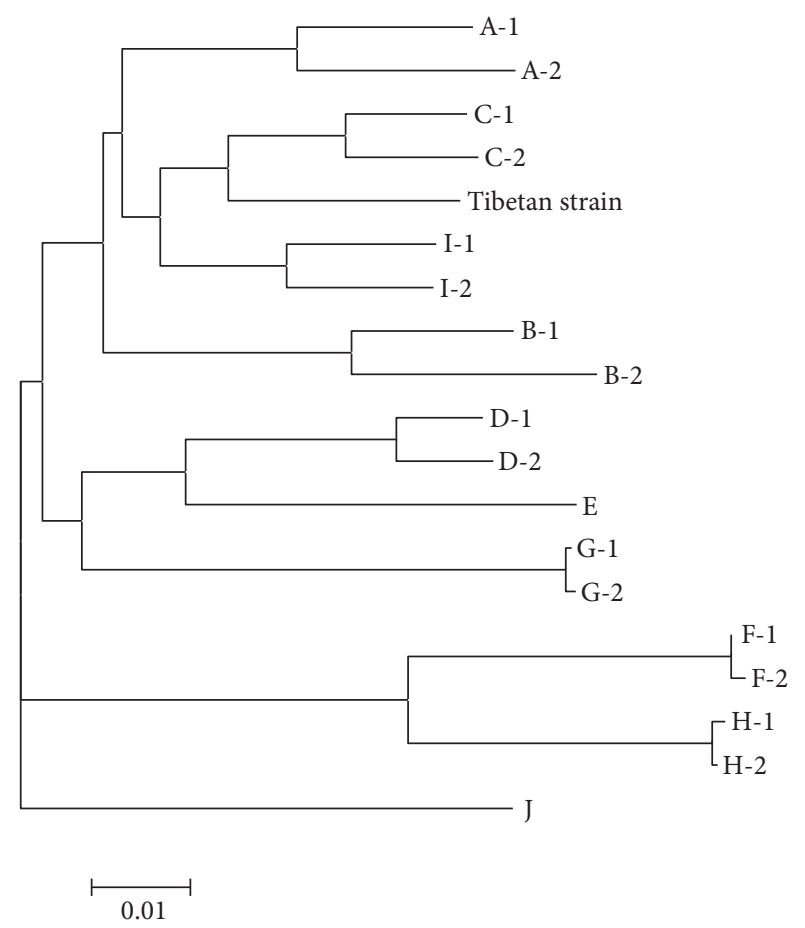

FIgure 2: Phylogenetic analysis of the selected Tibetan viral strain. Reference Sequences from NCBI: genotype A: A-1(AM282986.1) and A-2(JN182318.1); genotype B: B-1(AB368295.1) and B2(EF494382.1); genotype C: C-1(AB368296.1) and C-2(AB368297.1); genotype D: D-1(AM422939.1) and D-2(AB554024.1); genotype E: E(FN594748.1); genotype F: F-1(AB036909.1) and F-2(AB036907.1); genotype G: G-1(AP007264.1) and G-2(AB625342.1); genotype $\mathrm{H}$ : H-1(AB275308.1) and H-2(AB179747.1); genotype I: I-1(FJ023659.1) and I-2(FJ023664.1); genotype J: J(AB486012.1). Full HBV genomic sequences were applied for phylogenetic analysis.

With the use of the replicative plasmid, the basic mechanism of clinical manifestations, virological features, and viral pathogenicity could be clarified. Furthermore, C/D recombinant should have included two subrecombinants, namely, $\mathrm{C} / \mathrm{D} 1$ and $\mathrm{C} / \mathrm{D} 2$. The recombinant region in $\mathrm{C} / \mathrm{D} 1$ strain covered preS2/S region (nt10-799), while C/D2 strain had a recombinant region from preS2/S to X regions (nt101499) [16]. In our study, the selected viral strain was more similar to C/D2 strain. Zhou et al. found that C/D1 strain infected patients would encounter lower serum bilirubin and lower frequency of G1896A mutation compared to C/D2 strain infected ones [24]. In the future, deep analysis into the $\mathrm{C} / \mathrm{D}$ recombinant should be expected.

\section{Materials and Methods}

4.1. Study Materials. Serum samples were collected from Han and Tibetan outpatients consulted in West China Hospital of Sichuan University: 314 samples were from Han outpatients from Sichuan Province and 17 samples were from Tibetan outpatients from Tibet. The HBV replicative plasmid pHBV4.1(D) and the vector plasmid pUC18 were reserved in our lab. Competent bacterium E. coli $\mathrm{DH} 5 \alpha$ was purchased from Takara Bio(Dalian) as well as LATaq PCR Amplification Kit, restriction endonuclease Hind III, Pst I, Xba I, and T4 DNA ligase. Viral DNA extraction kit was purchased from BioTeke (Beijing). SPF grade male BALB/c mice at age of 7-9 weeks and weight of 18-20 g were provided by West China Laboratory Animals Center of Sichuan University.

\subsection{Study Procedure}

4.2.1. Clinical Data Collected and Analysis. Clinical data of patients who provided these serum samples were collected retrospectively from the electronic medical records in the hospital. The clinical manifestation and virological features were analyzed and compared between Han patients and Tibetan patients. Then, these data in C/D recombinant infected patients were analyzed and compared with that of all patients to learn whether the $\mathrm{C} / \mathrm{D}$ recombinant had some unique clinical manifestation or virological features.

\subsubsection{Serum Treatment and Viral Genotype Determination.} One aliquot of the collected serum sample was sent to a commercial laboratory (Kingmed Co., Sichuan) for HBV genotype determination. The laboratory used $200 \mu \mathrm{L}$ serum for extracting viral nucleic acid. Genotypes were determined by direct Sanger sequencing; the RT region of the viral genome was amplified on ABI 3130 Genetic Analyzer (South San Francisco, CA, USA) and analyzed in an alignment search tool (Chromas 2.23, Technelysium, South Brisbane, QLD, Australia) according to National Center for Biotechnology Information Genotyping Database. After acquiring the final result of genotypes, subgenotypes, or recombinants, the laboratory reported it to us. Since the C/D recombinant strain was identified, we used the other aliquot of the same serum sample for extracting viral DNA genome. In this procedure, we used the DNA extraction kit (BioTeke, Beijing). The extraction would then be used as template for PCR amplification.

4.2.3. Plasmid Construction. Because the 1.3 copies of $\mathrm{HBV}$ genomic DNA could not be acquired directly, we divided the amplified DNA into two different fragments, $2.4 \mathrm{~Kb}$ and $1.7 \mathrm{~Kb}$, at the $\mathrm{Xba} \mathrm{I}$ restriction site. The two fragments both crossed the gap in the minus strand of viral genome. Thus such division would form either a circular template without gap or a linearized template with double genomic DNA. To acquire such kind of templates, enough HBV full genomic DNA need to be amplified for subsequent ligation. However, the HBV genome is nonclosed circular and partially double-strands DNA; direct amplification of the full genome is difficult. Gunther method solved this difficulty and we applied it for our study [28]. To accomplish the amplification, a primer pair (Table 3), focusing on the gap of the minus strand as well as containing specifically DR1 (direct repeat sequence 1) and the same restriction nuclease site (Sap I), was designed. The amplified DNA was then cyclized or double ligated. Then another two pairs of primers (also in Table 3) were designed to amplify the two fragments of $1.7 \mathrm{~Kb}$ and $2.4 \mathrm{~Kb}$. Restriction sites of Hind III and Xba I were designed into the primer pair for $2.4 \mathrm{~Kb}$ fragment, while Xba I and Pst I 

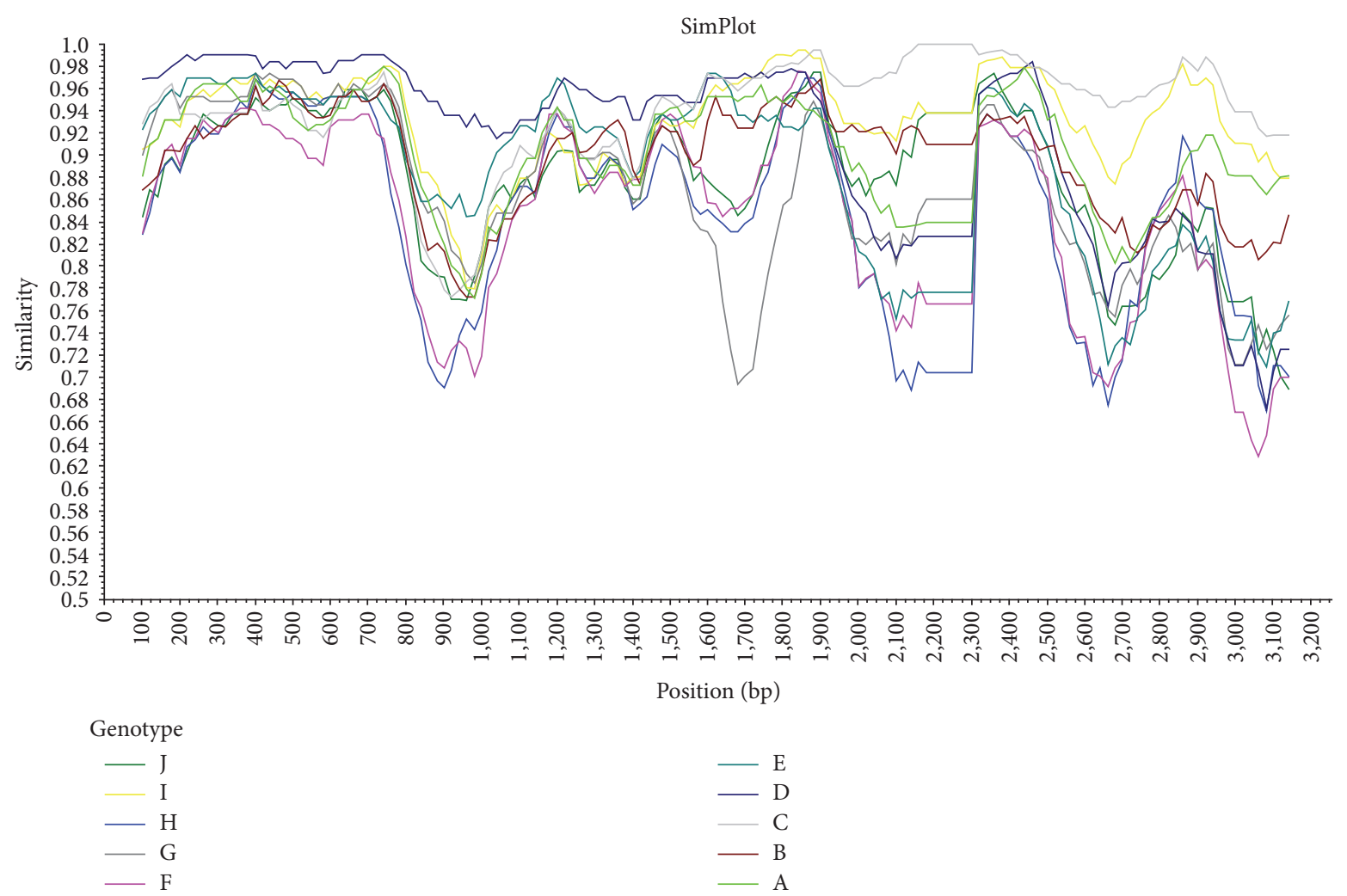

FIgURE 3: Recombination analysis of the selected Tibetan viral strain. Phylogenetic analysis data were also used as alignment data. The sequence similarity of the selected Tibetan strain to viral strains of all ten genotypes from A to J was analyzed. The position of nucleotide bases was shown in the abscissa and the similarity of the selected Tibetan strain to reference strains was shown in the ordinate. The numbering of HBV genome started from the restriction site of EcoR I downstream of preS2 initiation codon. Each curve represented one genotype and it showed the variation of sequence similarity between the selected Tibetan strain and the chosen reference sequence at each base site in the full genome. As shown, the dark blue curve, which was at the top left, implied that the fragment (nt1-1480) of the Tibetan HBV genome covering preS2/S region and X region had the highest similarity to genotype D. And the light grey curve, which was at the top right, implied that the rest of the genome had the highest similarity to genotype C.

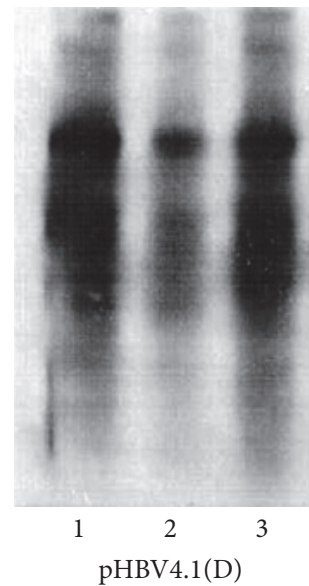

pHBV4.1(D)

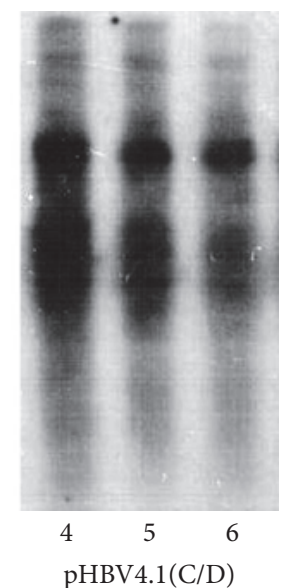

(a)

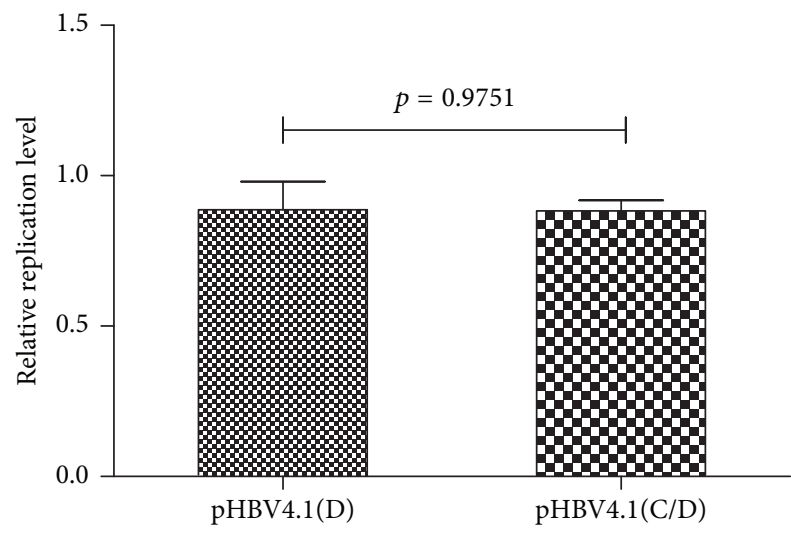

(b)

FIGURE 4: HBV replication intermediates in mouse models established with pHBV4.1(D) and pHBV(C/D). Mice were injected hydrodynamically with $10 \mu \mathrm{g}$ pHBV4.1(D) orpHBV4.1(C/D) and mouse livers were harvested 3 days after modeling. HBV replication intermediates in the liver were detected through DNA (Southern) filter hybridization (a). The image of DNA (Southern) filter hybridization was imported into Quantity One (Bio-Rad Laboratories, Life Science) and analyzed. The module of Volume Rect Tool was applied to generate rectangles covering all the bands in each lane and density of delineated bands was qualified and compared (b). The two plasmids possessed similar replication capacity in vivo. 
TABLE 3: Primers for construction of $\mathrm{pHBV} 4.1 \mathrm{C} / \mathrm{D}$ with viral genome in serum sample.

\begin{tabular}{|c|c|}
\hline \multicolumn{2}{|c|}{ Primers for amplification of HBV genome } \\
\hline Upstream & 5'-CCGGAAAGCTTGAGCTCTTCTTTTTCACCTCTGCCTAATCA-3' \\
\hline Downstream & $5^{\prime}$-CCGGAAAGCTTGAGCTCTTCAAAAAGTTGCATGGTGCTGG-3' \\
\hline \multicolumn{2}{|c|}{ Primers for amplification of $2.4 \mathrm{~Kb}$ fragment } \\
\hline Upstream & $5^{\prime}$-CCTGCTTTAATGCCTTTGTATGC-3' \\
\hline Downstream & $5^{\prime}$-TCCACCACGAGTCTAGACTCTGTGG-3' \\
\hline \multicolumn{2}{|c|}{ Primers for amplification of $1.7 \mathrm{~Kb}$ fragment } \\
\hline Upstream & $5^{\prime}$-CCACAGAGTCTAGACTCGTGGTGGA-3' \\
\hline Downstream & $5^{\prime}$-CGGTGTCGAGGAGATCTCGAATAGA-3' \\
\hline
\end{tabular}

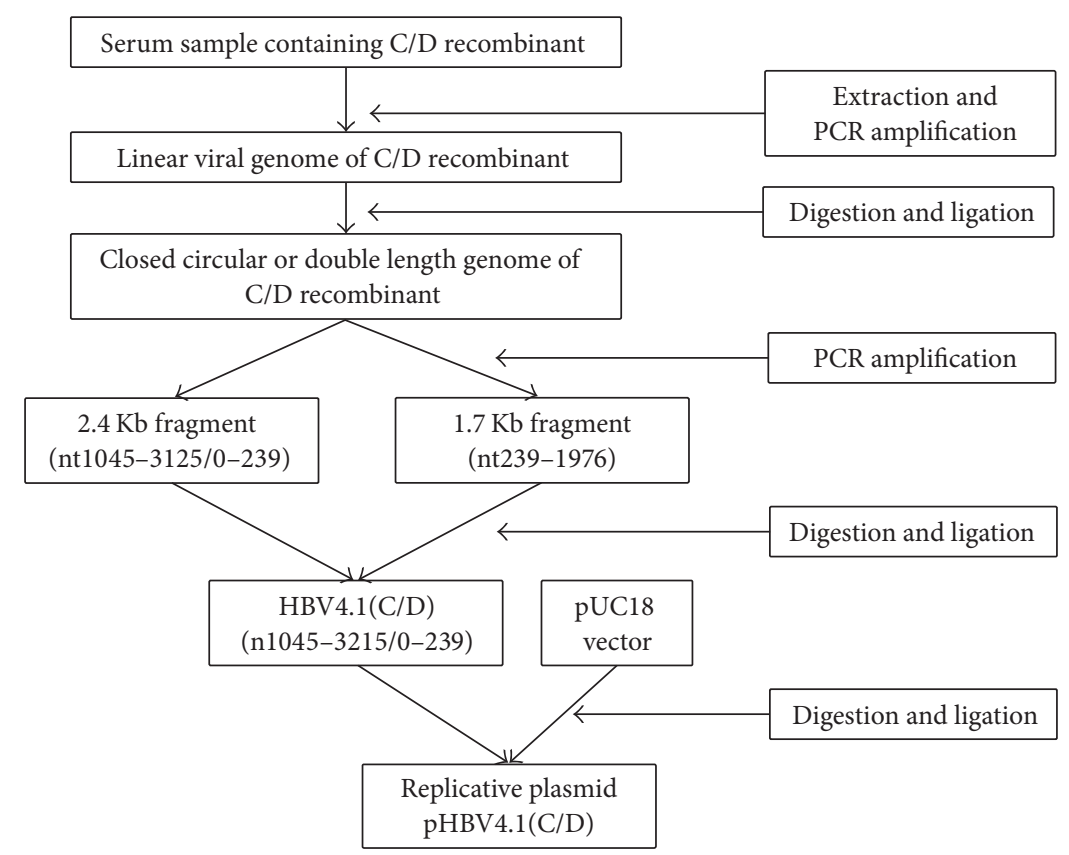

FIGURE 5: Construction procedure of clonal plasmid containing 1.3 copies of HBV genomic DNA of C/D recombinant.

were designed into the primer pair for $1.7 \mathrm{~Kb}$. After digestion and gel extraction, these two fragments were ligated and inserted into the clonal plasmid vector pUC18 between the DNA restriction sites Hind III and Pst I. pUC18 is a popular vector molecule, it helped to quickly distinguish the recombinants from nonrecombinants based on colonies' color [29]. Then the monoclonal bacteria containing the recombinant plasmid were picked up for plasmid extraction. Both restriction nuclease digestion and full genome amplification with extracted plasmid were applied to confirm a successful plasmid construction. The protocol was shown in Figure 5.

4.2.4. Genotype Verification and Recombinant Analysis. Direct sequencing of the amplification DNA of the extracted plasmid was used for final genotype verification. The sequencing data were input into Clustal X multiple sequence alignment program along with 18 reference genetic sequences from genotypes A to J acquired in "Basic Local Alignment Search Tool" (U.S. National Library of Medicine) for alignment. The selected reference genomic sequences were as follows: genotype A: A-1(AM282986.1) and A-2(JN182318.1); genotype B: B-1(AB368295.1) and B-2(EF494382.1); genotype C: C-1(AB368296.1) and C-2(AB368297.1); genotype D: D1(AM422939.1) and D-2(AB554024.1); genotype E: E(FN594748.1); genotype F: F-1(AB036909.1) and F-2(AB036907.1); genotype G: G-1(AP007264.1) and G-2(AB625342.1); genotype H: H1(AB275308.1) and H-2(AB179747.1); genotype I: I-1(FJ023659.1) and I-2(FJ023664.1); and genotype J: J(AB486012.1). Clustal $\mathrm{X}$ would generate an alignment file. Then the file was input into MEGA 5.10. Phylogenetic analysis was carried out in MEGA 5.10 with the Kimura two-parameter matrix model and neighboring-joining method and bootstrap resampling were performed 1,000 times. As a result, a phylogenetic tree was established and evolution of the to-be-identified HBV genome could be learned. Afterwards, the above alignment file was input into Simplot 3.5.1 to process the recombination analysis. Identity between the to-be-identified HBV genome and references sequences was calculated. The bootscan window sizes were 200 bases and the step size was 20 bases with 100 replicates [30]. 
4.2.5. Replication Capacity Detection. Hydrodynamic HBV replicative mouse model was used to verify the replication activity of the newly constructed plasmid in vivo. A solution of $10 \mu \mathrm{g}$ naked replicative plasmid with a volume over total blood volume of a mouse (about $8 \%$ of weight) was transferred into $\mathrm{BALB} / \mathrm{C}$ mouse via the tail vein within 5 to 8 seconds [26]. Circular congestion resulted in transient heart failure and backflow of the liquid from postcava to liver. Fast injection decreased the contact time of plasmid to nuclease in serum. Thus sufficient plasmid entered hepatic sinusoid and was engulfed by hepatocytes. Such mouse model provided transient viral DNA replication, RNA transcription, and protein expression lasting for 10 days with a peak around day 3. It fulfilled the requirement of studies in viral biological characteristics [31]. Besides the newly constructed plasmid; the existing plasmid pHBV4.1(D) from our lab was used as the control group. Three days after modeling, the mouse sera were collected for viral DNA extraction with the same extraction kit mentioned before. And the mouse liver was harvested to extract HBV replication intermediates.

With the same primer pair and reaction condition, full HBV genomic DNA in mouse serum was amplified. Then the amplified products were sequenced and analyzed in BLAST again to identify the genotype.

Viral replication intermediates were isolated according to previously descried methods [26]: one hundred and twenty micrograms of liver tissue in each sample was lysed for isolation and the isolated DNA replication intermediates were dissolved in $30 \mu \mathrm{L} 10 \mathrm{mmol} / \mathrm{L}$ tris hydrochloride ( $\mathrm{pH} \mathrm{8.0)}$ and $1 \mathrm{mmol} / \mathrm{L}$ EDTA. In the protocols of $\mathrm{HBV}$ replication intermediates extraction, DNase was added to digest contaminated host genomic DNA and injected plasmid. We added $24 \mu \mathrm{L}$ DNase (D4527, Sigma-Aldrich, USA) solution with a concentration of $5 \mathrm{mg} / \mathrm{mL}$ to acquire a working concentration of $200 \mu \mathrm{g} / \mathrm{mL}$ in each sample lysed from $0.12 \mathrm{~g}$ mouse liver powder. And with introduction of DNase digestion during the extraction procedure, the input plasmid DNA was removed and the isolated nucleotides were purified replication intermediates. DNA (Southern) filter hybridization was performed with the $30 \mu \mathrm{L}$ viral replication intermediates. Filter was probed with DIG Luminescent Detection Kit (Roche Applied Science) labeled full-length HBV genomic DNA (genotype D) and the detected replication intermediates were qualified in image analysis system (Quantity One, Bio-Rad Laboratories, Life Science). We collected Southern Blot images through an equal time interval of 5 minutes after the filter was infiltrated by luminol substrate solution. And the collection time usually lasted for 90 minutes. All these procedures were automatically implemented in ChemiDoc ${ }^{\mathrm{TM}}$ MP Imaging System (Bio-Rad, USA). Through such method, we could acquire a series of gradually enhanced images. And the proper images were picked up.

Three independent experiments were conducted, and totally six mice were modeled in each experiment: 3 in the pHBV4.1(D) group and 3 in the pHBV4.1(C/D) group.

4.3. Statistical Analysis. Experimental data were captured with Quantity One from photographic film. All data were analyzed with SPSS 18.00. Enumeration data were described by percentage and analyzed with $\chi^{2}$ test. Measurement data were described by mean \pm standard deviation or median (interquartile range) according to their distribution characteristics and analyzed with $t$-test or $Z$ test.

4.4. Ethics Statements. Experiments were performed in compliance with relevant laws and institutional guidelines and in accordance with the ethical standards of the Declaration of Helsinki. All serum samples were acquired with written informed consent under the permission from West China Hospital Ethics Committee. Animal studies were approved by Laboratory Animal Ethics Committee of Sichuan University (Project Identification Code: 2012-76).

\section{Conclusion}

In conclusion, our study found that in Sichuan Han patients from Southwest China, genotype $B$ and $C$ viral strains were prevalent. And this distribution was consistent with the geographic distribution of $\mathrm{HBV}$ genotypes in China. Meanwhile, our study found that $\mathrm{C} / \mathrm{D}$ recombinant strain was rare in Han patients from Sichuan Province, yet it was dominant in Tibetan patients from Tibet. Following this finding, an HBV replicative plasmid pHBV4.1(C/D) followed by an $\mathrm{HBV}$ replicative mouse model was constructed. An existing plasmid pHBV4.1(D) was set as the control group mouse model. Experiment results revealed that the $\mathrm{C} / \mathrm{D}$ recombinant replicated effectively at a similar level as genotype D viral strain did in the control group mouse model.

\section{Conflicts of Interest}

The authors declare no conflicts of interest.

\section{Authors' Contributions}

Hong Tang conceived the study, provided fund support, and revised the manuscript critically for important intellectual content. Taoyou Zhou and Cong Liu collected the serum samples. Lingyao Du, Miao Liu, and Xing Cheng implemented the experiments. Menghan Liu executed data analysis. Lingyao Du, Menghan Liu, and Taoyou Zhou participated in manuscript preparation. Lingyao Du draft the manuscript and revised it according to all authors' opinions. All authors have read and approved the final manuscript.

\section{Acknowledgments}

This work was supported by Science and Technology Project of Sichuan Province (no. 2015SZ0049).

\section{References}

[1] M. A. Purdy, G. Talekar, P. Swenson, A. Araujo, and H. Fields, "A new algorithm for deduction of hepatitis B surface antigen subtype determinants from the amino acid sequence," Intervirology, vol. 50, no. 1, pp. 45-51, 2006.

[2] A. Bartholomeusz and S. Schaefer, "Hepatitis B virus genotypes: Comparison of genotyping methods," Reviews in Medical Virology, vol. 14, no. 1, pp. 3-16, 2004. 
[3] S. Schaefer, "Hepatitis B virus taxonomy and hepatitis B virus genotypes," World Journal of Gastroenterology, vol. 13, no. 1, pp. 14-21, 2007.

[4] K. Tatematsu, Y. Tanaka, F. Kurbanov et al., "A genetic variant of hepatitis $B$ virus divergent from known human and ape genotypes isolated from a Japanese patient and provisionally assigned to new genotype J," Journal of Virology, vol. 83, no. 20, pp. 10538-10547, 2009.

[5] T. T. T. Huy, T. N. Trinh, and K. Abe, "New complex recombinant genotype of hepatitis B virus identified in Vietnam," Journal of Virology, vol. 82, no. 11, pp. 5657-5663, 2008.

[6] H. Yu, Q. Yuan, S.-X. Ge et al., "Molecular and phylogenetic analyses suggest an additional hepatitis B virus genotype "I"," PLoS ONE, vol. 5, no. 2, Article ID e9297, 2010.

[7] A. Kramvis, "Genotypes and genetic variability of hepatitis B virus," Intervirology, vol. 57, no. 3-4, pp. 141-150, 2014.

[8] A. Kramvis, K. Arakawa, M. C. Yu, R. Nogueira, D. O. Stram, and M. C. Kew, "Relationship of serological subtype, basic core promoter and precore mutations to genotypes/subgenotypes of hepatitis B virus," Journal of Medical Virology, vol. 80, no. 1, pp. 27-46, 2008.

[9] C. Mayerat, A. Mantegani, and P. C. Frei, "Does hepatitis B virus (HBV) genotype influence the clinical outcome of $\mathrm{HBV}$ infection?" Journal of Viral Hepatitis, vol. 6, no. 4, pp. 299-304, 1999.

[10] M.-W. Yu, S.-H. Yeh, P.-J. Chen et al., "Hepatitis B virus genotype and DNA level and hepatocellular carcinoma: A prospective study in men," Journal of the National Cancer Institute, vol. 97, no. 4, pp. 265-272, 2005.

[11] H. L.-Y. Chan, A. Y. Hui, M. L. Wong et al., "Genotype C hepatitis B virus infection is associated with an increased risk of hepatocellular carcinoma," Gut, vol. 53, no. 10, pp. 1494-1498, 2004.

[12] A. Aghakhani, R. Hamkar, N. Zamani et al., "Hepatitis B virus genotype in Iranian patients with hepatocellular carcinoma," International Journal of Infectious Diseases, vol. 13, no. 6, pp. 685-689, 2009.

[13] V. Thakur, R. C. Guptan, S. N. Kazim, V. Malhotra, and S. K. Sarin, "Profile, spectrum and significance of HBV genotypes in chronic liver disease patients in the Indian subcontinent," Journal of Gastroenterology and Hepatology, vol. 17, no. 2, pp. 165-170, 2002.

[14] H. Norder, A.-M. Couroucé, P. Coursaget et al., "Genetic diversity of hepatitis B virus strains derived worldwide: Genotypes, subgenotypes, and HBsAg subtypes," Intervirology, vol. 47, no. 6, pp. 289-309, 2004.

[15] M. Yousif and A. Kramvis, "Genotype D of hepatitis B virus and its subgenotypes: An update," Hepatology Research, vol. 43, no. 4, pp. 355-364, 2013.

[16] S. Ghosh, P. Banerjee, P. Deny et al., "New HBV subgenotype D9, a novel D/C recombinant, identified in patients with chronic HBeAg-negative infection in Eastern India," Journal of Viral Hepatitis, vol. 20, no. 3, pp. 209-218, 2013.

[17] M. R. Pourkarim, S. Amini-Bavil-Olyaee, F. Kurbanov, M. Van Ranst, and F. Tacke, "Molecular identification of hepatitis B virus genotypes/subgenotypes: Revised classification hurdles and updated resolutions," World Journal of Gastroenterology, vol. 20, no. 23, pp. 7152-7168, 2014.

[18] P. Simmonds and S. Midgley, "Recombination in the genesis and evolution of hepatitis B virus genotypes," Journal of Virology, vol. 79, no. 24, pp. 15467-15476, 2005.
[19] J.-H. Kao, "Hepatitis B viral genotypes: Clinical relevance and molecular characteristics," Journal of Gastroenterology and Hepatology (Australia), vol. 17, no. 6, pp. 643-650, 2002.

[20] F. Kurbanov, Y. Tanaka, K. Fujiwara et al., "A new subtype (subgenotype) Ac (A3) of hepatitis B virus and recombination between genotypes A and E in Cameroon," Journal of General Virology, vol. 86, no. 7, pp. 2047-2056, 2005.

[21] N. M. Araujo, "Hepatitis B virus intergenotypic recombinants worldwide: An overview," Infection, Genetics and Evolution, vol. 36, pp. 500-510, 2015.

[22] C. Cui, J. Shi, L. Hui et al., "The dominant hepatitis B virus genotype identified in Tibet is a C/D hybrid," Journal of General Virology, vol. 83, no. 11, pp. 2773-2777, 2002.

[23] X.-F. Sun, S.-J. Wen, H. Li et al., "Distribution of hepatitis B virus genotypes and subgenotypes among chronically infected patients in Xinjiang Uighur," Zhonghua Gan Zang Bing Za Zhi, vol. 17, no. 2, pp. 88-90, 2009.

[24] B. Zhou, L. Xiao, Z. Wang, E. T. Chang, J. Chen, and J. Hou, "Geographical and ethnic distribution of the HBV C/D recombinant on the Qinghai-Tibet Plateau," PLoS ONE, vol. 6, no. 4, Article ID e18708, 2011.

[25] L. G. Guidotti, B. Matzke, H. Schaller, and F. V. Chisari, "Highlevel hepatitis B virus replication in transgenic mice," Journal of Virology, vol. 69, no. 10, pp. 6158-6169, 1995.

[26] F.-J. Liu, L. Liu, F. He et al., "Establishment and primary application of a mouse model with hepatitis B virus replication," World Journal of Gastroenterology, vol. 13, no. 40, pp. 5324-5330, 2007.

[27] L. Shen, W. Yin, H. Zheng et al., "Molecular epidemiological study of hepatitis B virus genotypes in Southwest, China," Journal of Medical Virology, vol. 86, no. 8, pp. 1307-1313, 2014.

[28] S. Gunther, B.-C. Li, S. Miska, D. H. Kruger, H. Meisel, and H. Will, "A novel method for efficient amplification of whole hepatitis B virus genomes permits rapid functional analysis and reveals deletion mutants in immunosuppressed patients," Journal of Virology, vol. 69, no. 9, pp. 5437-5444, 1995.

[29] J. Vieira and J. Messing, "The pUC plasmids, an M13mp7derived system for insertion mutagenesis and sequencing with synthetic universal primers," Gene, vol. 19, no. 3, pp. 259-268, 1982.

[30] Q. Zhang, Y. Liao, J. Chen et al., "Epidemiology study of HBV genotypes and antiviral drug resistance in multi-ethnic regions from Western China," Scientific Reports, vol. 5, no. 1, 2015.

[31] Z. Gao, F.-J. Liu, L. Liu et al., "Application of hepatitis B virus replication mouse model," World Journal of Gastroenterology, vol. 16, no. 16, pp. 1979-1985, 2010. 

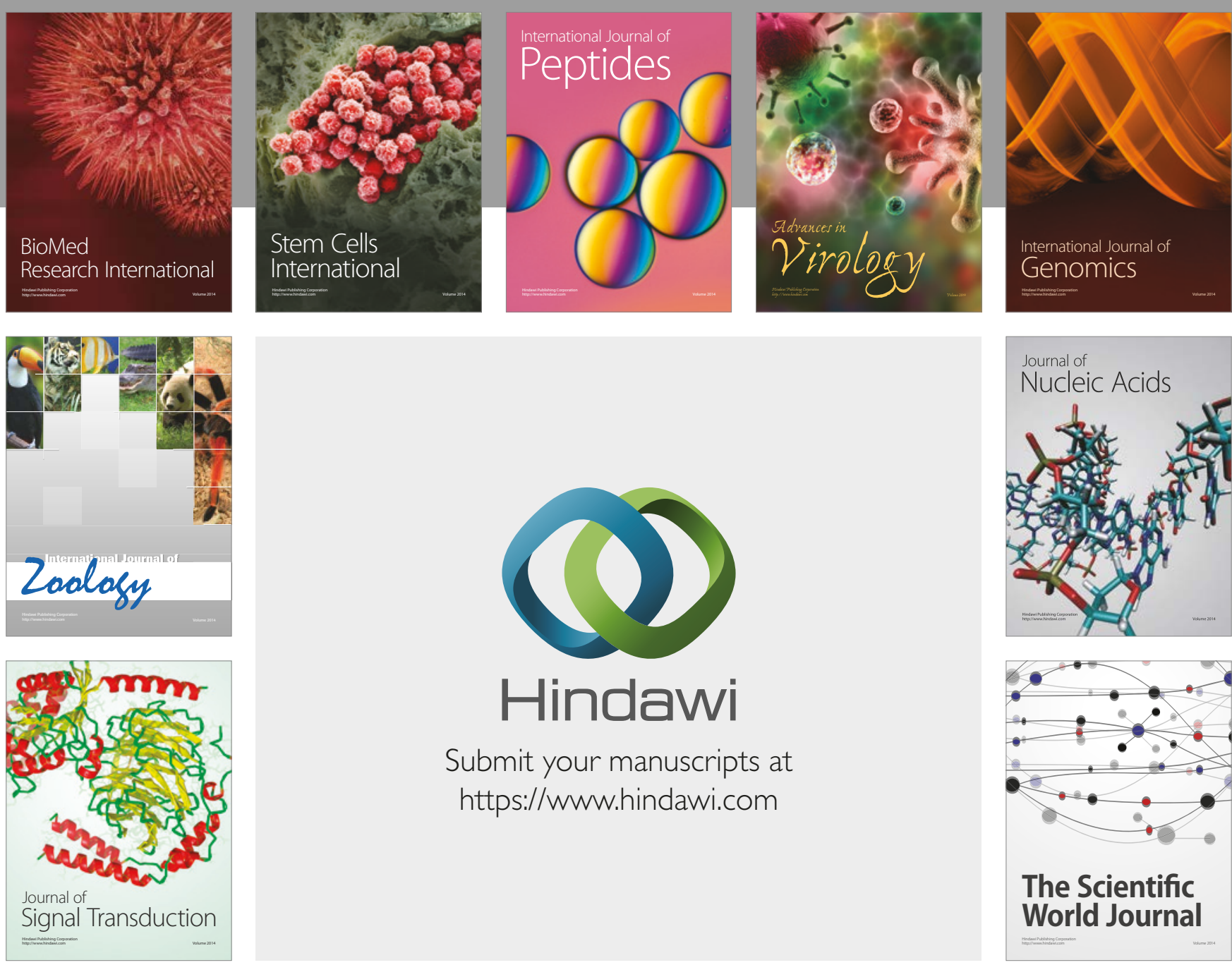

Submit your manuscripts at

https://www.hindawi.com
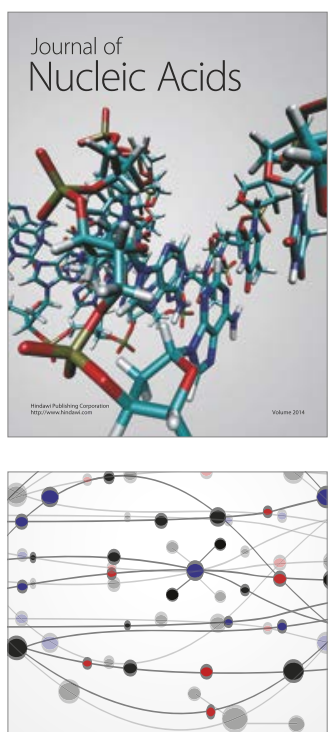

The Scientific World Journal

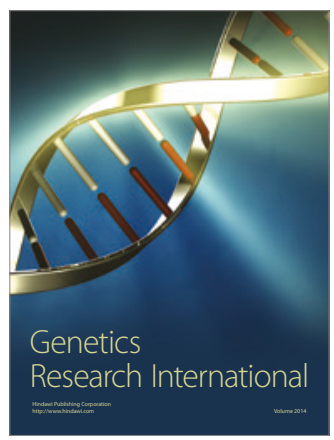

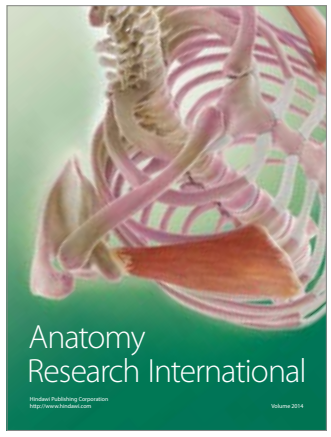

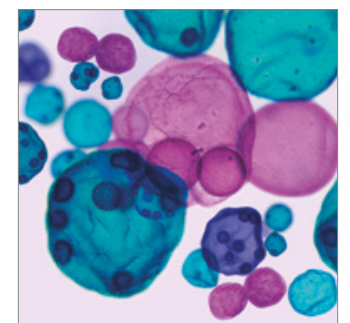

International Journal of Microbiology
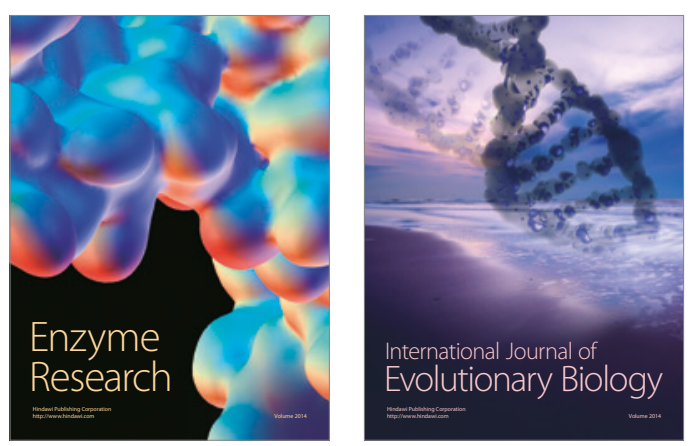
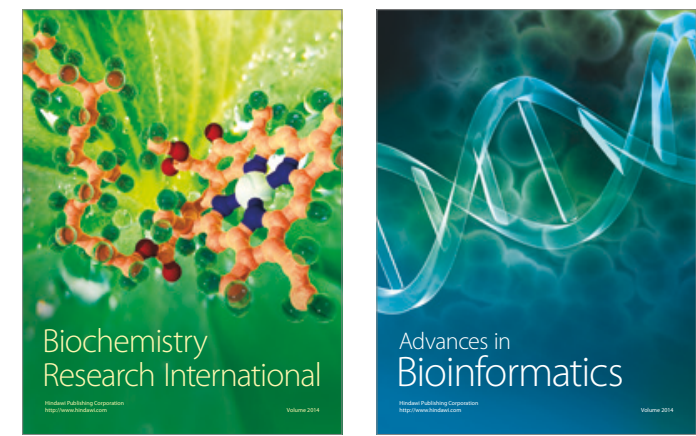

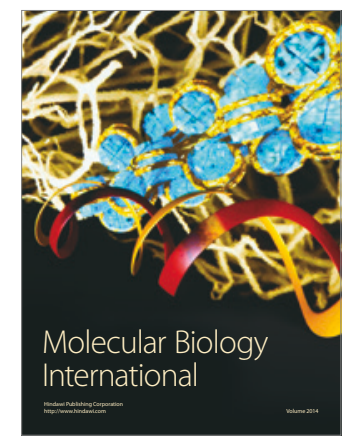

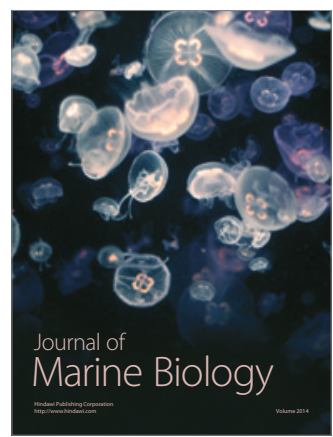

